\title{
Direct Numerical Simulation Guidance for Thorpe Analysis to Obtain Quantitatively Reliable Turbulence Parameters
}

\author{
LING WANG \\ GATS, Boulder, Colorado \\ MARVIN A. GELLER \\ Stony Brook University, State University of New York, Stony Brook, New York \\ DAVID C. FRITTS \\ GATS, Boulder, Colorado
}

(Manuscript received 8 December 2018, in final form 10 September 2019)

\begin{abstract}
Thorpe analysis has been used to study turbulence in the atmosphere and ocean. It is clear that Thorpe analysis applied to individual soundings cannot be expected to give quantitatively reliable measurements of turbulence parameters because of the instantaneous nature of the measurement. A critical aspect of this analysis is the assumption of the linear relationship $C=L_{O} / L_{T}$ between the Thorpe scale $L_{T}$, derived from the sounding measurements, and the Ozmidov scale $L_{O}$. It is the determination of $L_{O}$ that enables determination of the dissipation rate of turbulence kinetic energy $\varepsilon$. Single atmospheric and oceanic soundings cannot indicate either the source of turbulence or the stage of its evolution; different values of $C$ are expected for different turbulence sources and stages of the turbulence evolution and thus cannot be expected to yield quantitatively reliable turbulence parameters from individual profiles. The variation of $C$ with the stage of turbulence evolution is illustrated for direct numerical simulation (DNS) results for gravity wave breaking. Results from a DNS model of multiscale initiation and evolution of turbulence with a Reynolds number Re (which is defined using the vertical wavelength of the primary gravity wave and background buoyancy period as length and time scales, respectively) of 100000 are sampled as in sounding of the atmosphere and ocean, and various averaging of the sounding results indicates a convergence to a well-defined value of $C$, indicating that applying Thorpe analysis to atmospheric or oceanic soundings and averaging over a number of profiles gives more reliable turbulence determinations. The same averaging study is also carried out when the DNS-modeled turbulence is dominated by turbulence growing from the initial instabilities, when the turbulence is fully developed, when the modeled turbulence is decaying, and when the turbulence is in a still-later decaying stage. These individual cases converge to well defined values of $C$, but these values of $C$ show a large variation resulting from the different stages of turbulence evolution. This study gives guidance as to the accuracy of Thorpe analysis of turbulence as a function of the number of profiles being averaged. It also suggests that the values of $C$ in different environments likely depend on the dominant turbulence initiation mechanisms and on the Reynolds number of the environment.
\end{abstract}

\section{Introduction}

The direct calculation of the turbulence kinetic energy dissipation rate $\varepsilon$ in the oceans or atmosphere requires very high-resolution measurements to obtain the velocity shears at turbulence scales. This has led researchers to seek methods to calculate $\varepsilon$ from coarser measurements. One method introduced by Lübken

Corresponding author: Ling Wang, lwang@gats-inc.com
(1992; Lübken et al. 1993; Lübken 1997) is to derive a spatial spectrum for density measurements and perform a best fit to the transition from a $k^{-5 / 3}$ inertial range, where $k$ is wavenumber, to a Heisenberg (1948) or Tatarskii (1971) viscous range spectrum to determine the inner scale of turbulence, from which $\varepsilon$ can be estimated. More recently, velocity spectrum from the Leibniz Institute Turbulence Observations in the Stratosphere (LITOS) measurements are used to fit to those theoretical spectra to estimate $\varepsilon$ by the Lübken 
group (e.g., Schneider et al. 2015). Deriving such a spectrum from measurements requires very high resolution (a few millimeters or better in the stratosphere) measurements of winds and/or temperatures. Therefore, LITOS is a relatively expensive instrument, and thus has been deployed at only a few locations and times (Schneider et al. 2015, 2017).

Thorpe (1977) introduced a method to derive turbulence parameters from ocean soundings. Basically, his method was to examine oceanic density profiles for regions that were convectively unstable, that is to say, had greater densities overlying lesser densities. The profile is then sorted so that the profile is stable, that is to say, with greater densities underlying lesser densities. In this sorting process, each vertical displacement is referred to as a Thorpe displacement, and the root-mean-square (RMS) of the displacements over an unstable region is referred to as the Thorpe scale $L_{T}$. Conceptually, the Thorpe scale represents the vertical scale of the density overturning that initiates the turbulence. Deriving turbulence parameters from the Thorpe scale then requires relating it to the Ozmidov scale $L_{O}$, which is the largest length scale of turbulence before the effects of stratification are felt and is defined by

$$
L_{O}=\left(\varepsilon / N^{3}\right)^{1 / 2},
$$

where $N$ is the Brunt-Väisälä frequency and $\varepsilon$ is the turbulence kinetic energy dissipation rate. Thus, $\varepsilon$ can be determined once $L_{O}$ and $N$ are known. What remains, however, is determining $L_{O}$ from $L_{T}$, determined by the sorting process applied to the measured density profile.

Dillon (1982) examined a number of microstructure profiles in different water bodies and determined $\varepsilon$ by fitting the measured spectra to theoretical turbulence spectra, thus determining $L_{O}$ through Eq. (1). He also determined the Thorpe scales in these profiles by the previously described sorting process. $\mathrm{He}$ then determined the linear coefficient $C$, relating $L_{O}$ and $L_{T}$ via

$$
L_{O}=C L_{T}
$$

for each profile. Dillon (1982) found that the average value of $C$ over many profiles was 0.8 . Both Thorpe (1977) and Dillon (1982) pointed out that this was only true as an average over many profiles. This is obvious from inspection of Dillon's (1982) Table 1.

Clayson and Kantha (2008) pointed out that Thorpe analysis could be used on high-resolution radiosonde data, which with the advent of GPS geolocation, recorded data every second (in the U.S. data, at least), thus allowing turbulence parameters to be extracted. Based on their own and others' turbulence measurements, Clayson and Kantha (2008) adopted a value of $C=0.55$, but they noted large scatter in relating $L_{O}$ to $L_{T}$, implying that a single value of $C$ should only apply to an ensemble of measurements. Wilson et al. (2011) refined these methods to remove spurious unstable layers that were likely due to noise in the radiosonde soundings, and Wilson et al. (2013) presented methods that corrected the Thorpe analysis for moisture effects.

In summary then, in recent years, methods have been developed to characterize turbulent layers from highresolution operational radiosonde soundings with the realization that one cannot get quantitatively reliable estimates of turbulence parameters for individual turbulent layers in soundings, although detailed analysis of direct numerical simulation (DNS) results like in this study can provide guidance on the error bounds of these estimates.

These results have been reinforced by both numerical models of atmospheric turbulence and by comparison between LITOS measurements and Thorpe analysis of radiosonde data, where $L_{O}$ is determined by fitting spectra from LITOS wind measurements to theoretical spectra (Schneider et al. 2015). Their Fig. 5 shows a wide variation of the relation between $L_{O}$ and $L_{T}$, and their Fig. 8 showed that using the value of $L_{O}$ inferred by the linear relation between $L_{O}$ and $L_{T}$ led to many-ordersof-magnitude errors in estimating $\varepsilon$. Smyth and Moum (2000) performed DNS of Kelvin-Helmholtz instability (KHI) in layers of localized stratification and shear and found that the ratio between $L_{O}$ and $L_{T}$ depended on the stage of flow evolution and thus could be used as an indicator of the age of a KHI induced turbulence event. Fritts et al. (2016) showed results from DNS of turbulence resulting from the interaction between a single initial gravity wave (GW) and an initial oscillatory mean fine-structure shear with a vertical scale of one-fifth of the primary GW vertical wavelength for a Reynolds number $\operatorname{Re}=\lambda_{z}^{2} / T_{b}=100000$, where $\nu$ is kinematic viscosity, $\lambda_{z}$ is the vertical wavelength of the initial $\mathrm{GW}$, and $T_{b}=2 \pi / N$ (where $N$ is the background BruntVäisälä frequency), that is, the buoyancy period. Note that the corresponding peak turbulent Reynolds number, which is defined as $\mathrm{TKE}^{2} /(\varepsilon v)$, is $\sim 3000$ when the volume-averaged turbulent kinetic energy (TKE) and $\varepsilon$ are used. They compared model results with various measurements, which indicated that their modeled turbulence closely resembled observed atmospheric turbulence, and they also compared their values of $L_{O}$, derived from direct calculation of $\varepsilon$, with values of $L_{T}$, obtained by Thorpe analysis of the modeling results. Their Fig. 14 showed that the relation between $L_{O}$ and $L_{T}$ varied greatly depending on the type and stage of 
evolution of the various turbulent events in their model. Furthermore, the domain-averaged value of $C\left(=L_{O} / L_{T}\right)$ showed substantial time variation as the various turbulence events in the model domain evolved.

The purpose of this paper is to explore the results of Fritts et al. (2016) to see how averaging over many turbulence events leads to a convergence toward a single value of $C$ for a given stage in the flow evolution, thereby suggesting what magnitudes of errors may be expected in estimating $C$ and $\varepsilon$ by applying Thorpe analysis and adopting a single value for $C$. The remainder of this paper is organized as follows. Section 2 presents analysis of GW breaking events to see how $C$ evolves in time for those events. The purpose of this analysis is to get an idea of how $C$ evolves as the turbulence develops from the initial instability, persists, and dies out. Section 3 presents results for various levels of averaging of turbulent events chosen randomly from the Fritts et al. (2016) results to see what errors are associated with various levels of averaging when a single value of $C$ is adopted. Section 4 presents the conclusions of this study.

\section{Turbulence evolution for breaking GWs}

Fritts et al. (2016) identified three major turbulence initiation mechanisms in their DNS study. These were GW breaking, Kelvin-Helmholtz shear instability, and density-intrusion instability. Presumably, each of these instability sources of turbulence evolves differently, and might produce different ratios between $L_{O}$ and $L_{T}$. For instance, Scotti (2015) has noted that the ratio between $L_{O}$ and $L_{T}$ is different in convective instability induced turbulence than in turbulence produced from shear instability, with the value of $C$ being less for deep, convectively induced turbulence than for shear instability (see his Fig. 12). This is also consistent with comparisons of $\varepsilon$ derived from radar observations and Thorpe analysis of radiosonde data (Kohma et al. 2019).

Figures $1 \mathrm{a}$ and $1 \mathrm{~b}$ respectively show the time evolution of $L_{T}$ and $L_{O}$ from DNS models of GW breaking in a uniform environment. Similar DNS modeling, but with a lower-frequency primary $\mathrm{GW}$, is described in Fritts et al. (2009). In these models a nondimensional primary GW amplitude $a=\left|u^{\prime} / c\right|=1.1$ (where $u^{\prime}$ and $c$ are the GW horizontal perturbation velocity and phase speed, respectively) corresponds to a GW that is slightly unstable according to the linear criterion, whereas an $a=0.9 \mathrm{GW}$ is stable according to the linear criterion but becomes unstable as a result of the effects of nonlinearity. In Fig. $1, L_{T}$ and $L_{O}$ are scaled by $\lambda_{z}$, the vertical wavelength of the initial $\mathrm{GW}$, as defined above. Note that, for all plots (except for the DNS of $a=0.9$ and GW frequency of N/1.4) of the Thorpe scale,
$L_{T}$ starts at a large value and diminishes as the initial instability drives turbulence at smaller scales. For the DNS of $a=0.9$ and GW frequency of N/1.4, $L_{T}$ starts at an intermediate value and increases first before decreasing steadily. The Ozmidov scale starts at zero since, even though the instability exists, the turbulence has yet to be initiated. The $L_{O}$ then reaches a maximum, which is shown in all curves by the circles, and then diminishes as the turbulence decays and restratification occurs.

Figures $1 \mathrm{c}$ and $1 \mathrm{~d}$ respectively show the evolutions of $C$ and $\varepsilon ; \varepsilon$ is normalized by $\lambda_{z}^{2} / T_{b}^{3}$, where $T_{b}$ is the background buoyancy period, as defined above. The evolutions of $C$ in Fig. 1c are to be expected from the results of $L_{T}$ and $L_{O}$ described above. Note that $C$ reaches its maximum slightly after when $\varepsilon$ and $L_{O}$ are maximum; $C$ then levels off for a period of time as the turbulence decreases. These results suggest that radiosondes will pass through regions with widely varying values of $C$ but are very likely to pass through turbulent regions when $C$ is at its nearly constant value, since it stays near this value $4-5$ times as long as when $C$ is initially rapidly increasing while turbulence is growing or when turbulence is rapidly decreasing and the values of $\varepsilon$ are becoming very small. Note also that this period of near-constant $C$ coincides with turbulence decreasing from the largest values of $\varepsilon$. The results shown in Fig. 1 suggest that we might expect reasonably fast convergence toward the true values for $C$ and $\varepsilon$ as more averaging of measured unstable layers occurs.

Although Fig. 1 only relates to GW breaking as a source of turbulence, we expect similar behavior for turbulence arising from KHI and also for the densityintrusion source of turbulence. ${ }^{1}$ In this case of $\mathrm{GW}$ breaking, $C$ levels off for some time at a value of approximately 0.17 . We will return to this point later when we discuss expected differences in values of $C$ for different environments.

\section{Averaging results}

The multiscale DNS performed by Fritts et al. (2016) was a "spindown" flow initiated by a stable GW at a larger initial $\lambda_{z}$ and a stable oscillatory mean shear having a smaller initial $\lambda_{z}$. The superposition of these initial fields caused several types of instabilities, including local GW breaking, KHI, and intrusions having scales and intensities that decreased with time. As in the GW breaking DNS discussed above, this multiscale

\footnotetext{
${ }^{1}$ Scotti's (2015) results for convective sources of turbulence suggest that this behavior occurs for the density-intrusion case.
} 

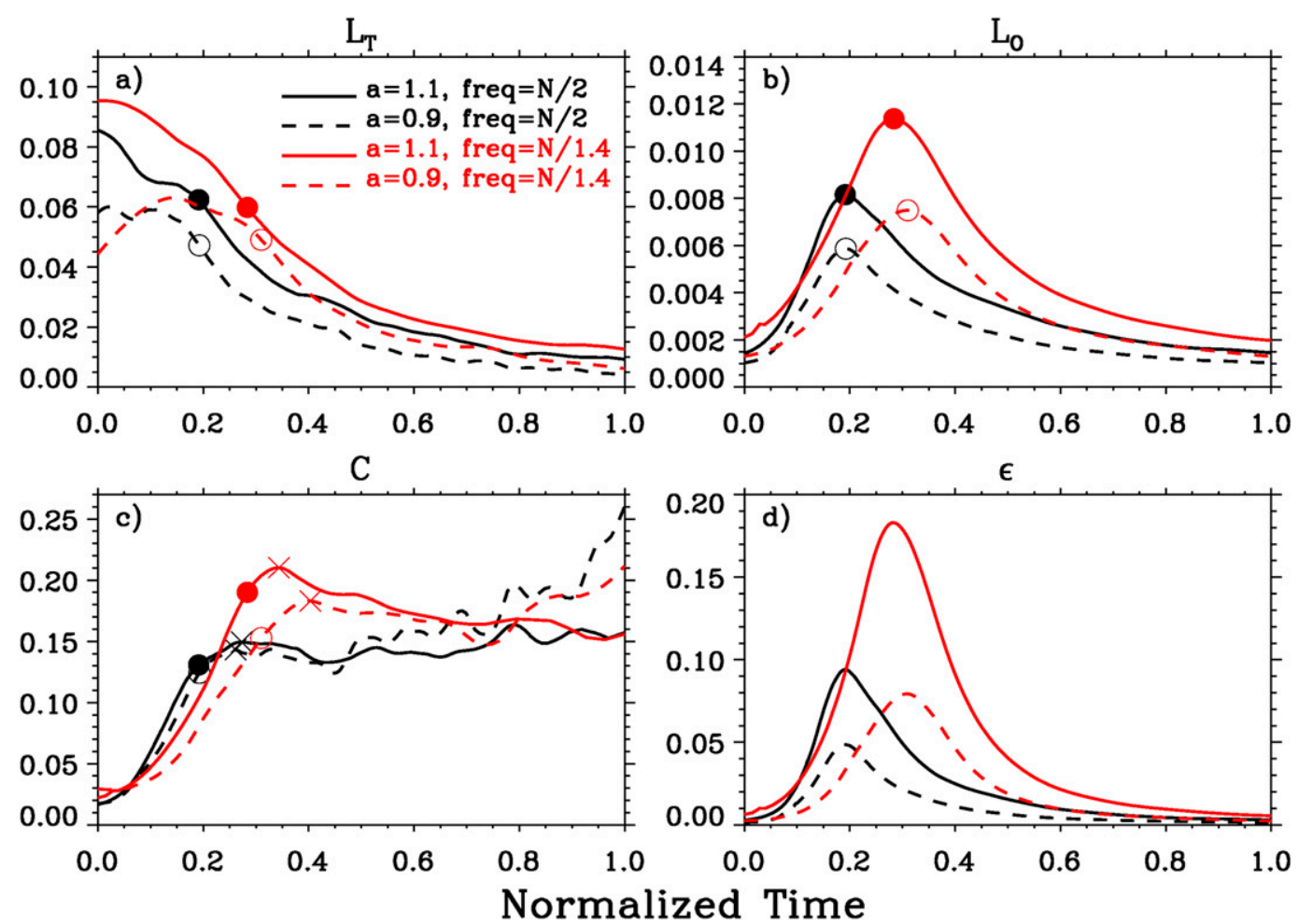

FIG. 1. Evolution of domain-averaged (a) $L_{T}$, (b) $L_{O}$, (c) $C$, and (d) $\varepsilon$ for four GW breaking DNS with an initial primary GW of nondimensional amplitude $a=0.9$ and 1.1 (dashed and solid lines, respectively) and intrinsic frequency of $N / 2$ and $N / 1.4$ (black and red lines, respectively), where $N$ is the background buoyancy frequency. Because of the disparate starting times and durations of active wave breaking and strong turbulence in these DNS, for easier comparison, the model times are normalized linearly such that 0 corresponds to the early stages of GW breaking, when $\varepsilon$ is $1 \%$ of its maximum value, and 1 is when $\varepsilon$ decreases back to $1 \%$ of its maximum value. Very early and late times when the flow was laminar are not shown here. Solid and open circles denote when $\varepsilon$ is at its maximum. The crosses in (c) indicate when $C$ reaches its initial maximum for each simulation. The DNS results are nondimensional. See the text for details.

DNS enables an evaluation of $L_{T}$ and $L_{O}$ from the onset of instabilities (with $L_{O}=0$ ) through the instability evolutions, the generation of turbulence, and its subsequent decay. As such, this DNS also enables an evaluation of the assessment of $L_{T}, L_{O}$, and $C$ for a more general multiscale flow such as we expect in the atmosphere based on the number of profiles employed for each field sampled.

We have explored the effects of averaging on the convergence of estimates of $C$ by constructing averages of $C$ from various numbers of profiles obtained at randomly selected times and locations within the multiscale DNS volumes between $7 T_{b}$ and $20 T_{b}$, when turbulence was well developed within the model domain [see the top panel of Fritts et al.'s (2016) Fig. 14]. To calculate $L_{T}$ for each profile from the multiscale DNS, we first calculate the Thorpe displacements $d^{\prime}$ from which we evaluated the largest absolute value of Thorpe displacement $d_{\max }^{\prime}$ for that profile. We then derive the local $L_{T}$ for a given vertical segment with a distance of $1.2 d_{\max }^{\prime}$ by calculating the RMS of $d^{\prime}$ within that segment. We repeat this calculation of local $L_{T}$ by sampling vertical segments of distance $1.2 d_{\max }^{\prime}$ continuously through the entire potential temperature profile and thus are able to obtain a vertical profile of $L_{T}$. Last, the profile average of $L_{T}$ is used to determine $C$ for that profile. We obtain $\varepsilon$, and hence $L_{O}$, directly from the respective velocity fields. Examples of the results of this procedure are shown in Fig. 2. As in Fig. 1, the results shown here are nondimensionalized using a basic length scale of $\lambda_{z}$ and time scale of $T_{b}$.

Figure 3 shows the values of the averages of $C$, as described above, over the indicated number of randomly selected profiles from the multiscale DNS simulation of Fritts et al. (2016), for profiles between $7 T_{b}$ and $20 T_{b}$. It is clear that there is much scatter within the determined values of $C$, but, as the number of profiles in the averaging increases, the values converge toward $C=0.53$. This value is considerably higher than the values of $C$ from the GW breaking simulations shown in Fig. 1. 


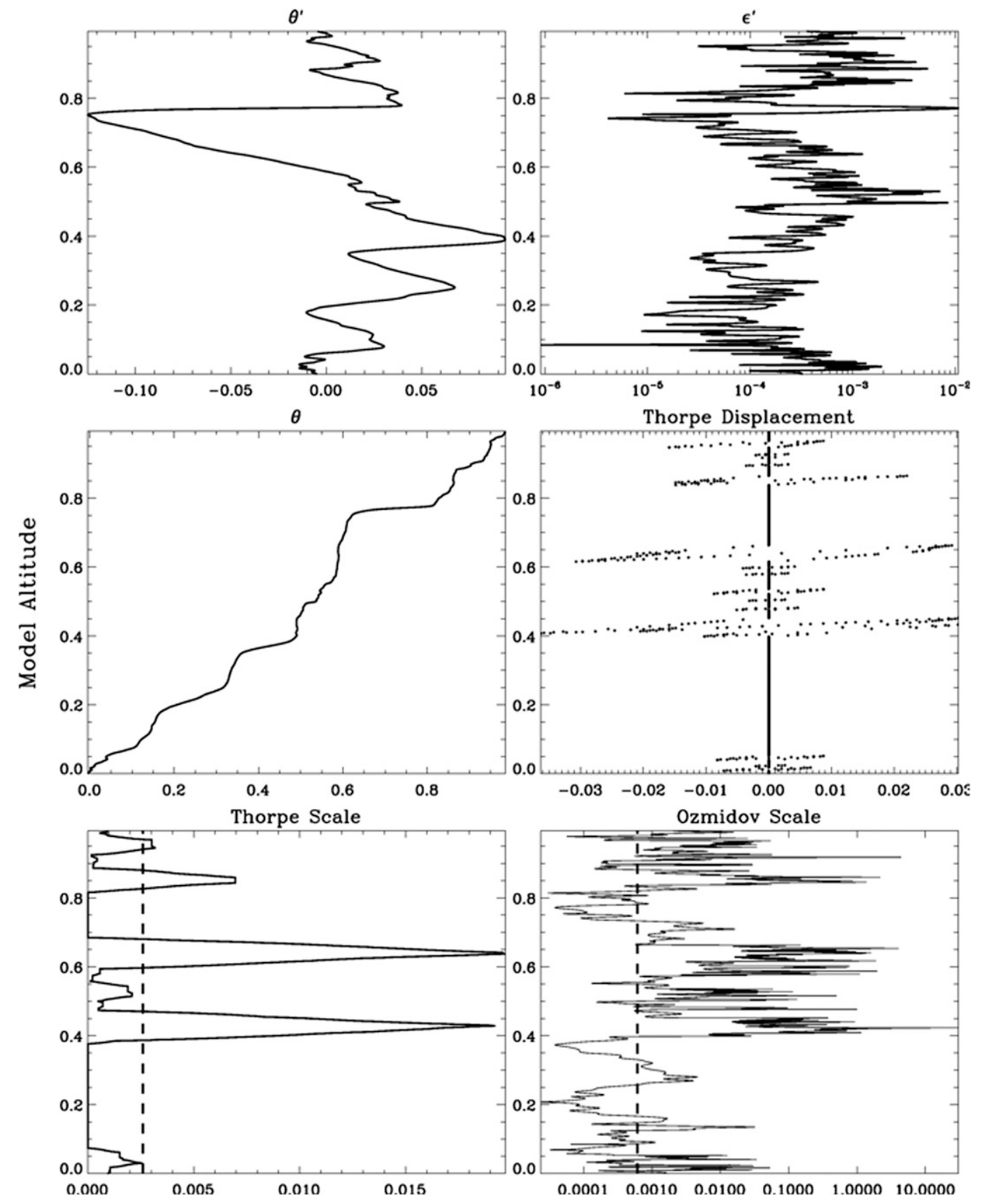

FIG. 2. Profiles of $\theta^{\prime}$ (potential temperature fluctuation), $\varepsilon, \theta$ (before sorting), $d^{\prime}$ (Thorpe displacements), $L_{T}$, and $L_{O}$ for a randomly selected profile at $11.26 T_{b}$ from the multiscale DNS modeling of Fritts et al. (2016); $C=L_{O} / L_{T}=0.23$ for this profile. The vertical black dashed lines indicate the average values of $L_{O}$ and $L_{T}$. All of the quantities here are nondimensional. See the text for details.

There are two possible reasons for this result. One is that local GW breaking in a multiscale flow is much weaker than strong GW breaking in a domain of comparable vertical scale. Weaker GW breaking events are generally less sustained in their peak turbulent stage and thus there is greater chance observing them in the decaying turbulence stage, when $C$ is large, despite active instability events elsewhere in the flow at these times. Second, KHI is a significant contributor in the multiscale DNS, and the values of $C$ that it contributes may be substantially higher than those from GW breaking. There is some support for this by examining Fig. 14 and Table 1 of Fritts et al. (2016). We explore the dependence on the stages of turbulence in more detail below. 


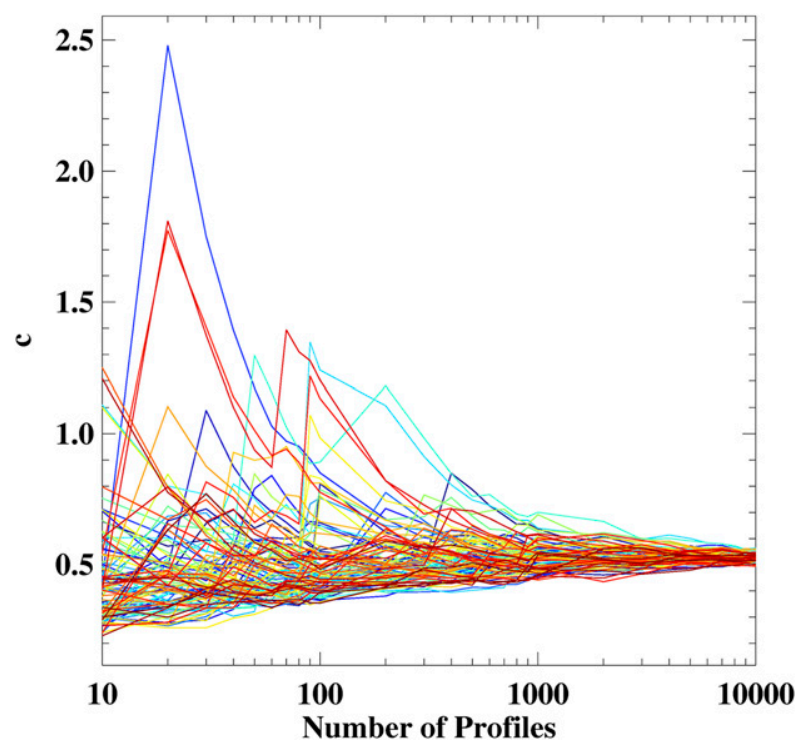

FIG. 3. Averages of $C=L_{O} / L_{T}$ from the indicated number of profiles through the multiscale DNS simulation of Fritts et al. (2016) at randomly selected times from $7 T_{b}$ to $20 T_{b}$. The Reynolds number for this simulation was 100000 . There are 100 colored lines, with each representing one realization of the random sampling of profiles for a given number of profiles ranging from 10 to 10000 .

By examining Fritts et al.'s (2016) Fig. 14, one sees a substantial variation in the domain-averaged value of $C$ at different times. As expected from both the results shown in Fig. 14 of Fritts et al. (2016) and implied by Fig. 1 in this paper, convergence to the smallest values for $C$ is expected for initially growing turbulence, while convergence to the largest values of $C$ is expected for the late stages of turbulence decay.

Figure 4 shows results by following the same procedure that was used in producing Fig. 3. Here, we take the interval from $7 T_{b}$ to $12 T_{b}$ as representative of turbulence in its growing phase due to initial instability sources; the interval from $12 T_{b}$ to $17 T_{b}$ as representative of well-developed turbulence with active sources and dissipation; the interval from $27 T_{b}$ to $32 T_{b}$ when decaying turbulence outweighs additional sources; and a final interval from $32 T_{b}$ to $40 T_{b}$ during which sources are very weak and turbulence is largely decaying. All four plots show similar convergence as averages are taken for an increasing number of profiles, but as expected the convergence at times of developing turbulence (left panel) approaches a smaller value of $C$ (about 0.21 ); at times of fully developed turbulence (left-center panel) the convergence is to a value for $C$ (about 0.58 ) that is close to that for $7-20 T_{b}$ (about 0.53 ); at times of decaying turbulence $\left(27-32 T_{b}\right)$, the convergence is to a much larger value (about 1.52), and at a still-later stage of turbulence decay $\left(32-40 T_{b}\right)$ the convergence is to a still-larger value (about 2.75). The implication is that the values of $C$ at the initial stage of turbulence initiation are about an order of magnitude less than those when turbulence is in its late stages of decay. One cautionary note is that time averages should be taken over a time period when the flow is not changing much to satisfy ergodicity. Although the flow of Fritts et al. (2016) is continually evolving, we believe the ergodicity assumption is generally satisfied for the intervals chosen here especially during the more turbulent stages.

Table 1 is an indicator of the errors associated with the various levels of averaging (taken from the $7-20 T_{b}$ plot in Fig. 3). In this table, we have also calculated the squares of the differences between the individual values of $C$ for different degrees of averaging. Taking the square root of these, we obtain the distribution of the absolute values of the differences between the individual determinations and the convergence value of $C$. The RMS of these differences is an indicator of the expected errors in determining $C$ for various degrees of averaging. If we expect the errors to be normally distributed and identify the absolute RMS with the standard deviation of the expected error, then we expect a determination of $C$ from averaging 100 profiles to be within about $66 \%$ of its true value; determination of $C$ from 1000 profiles
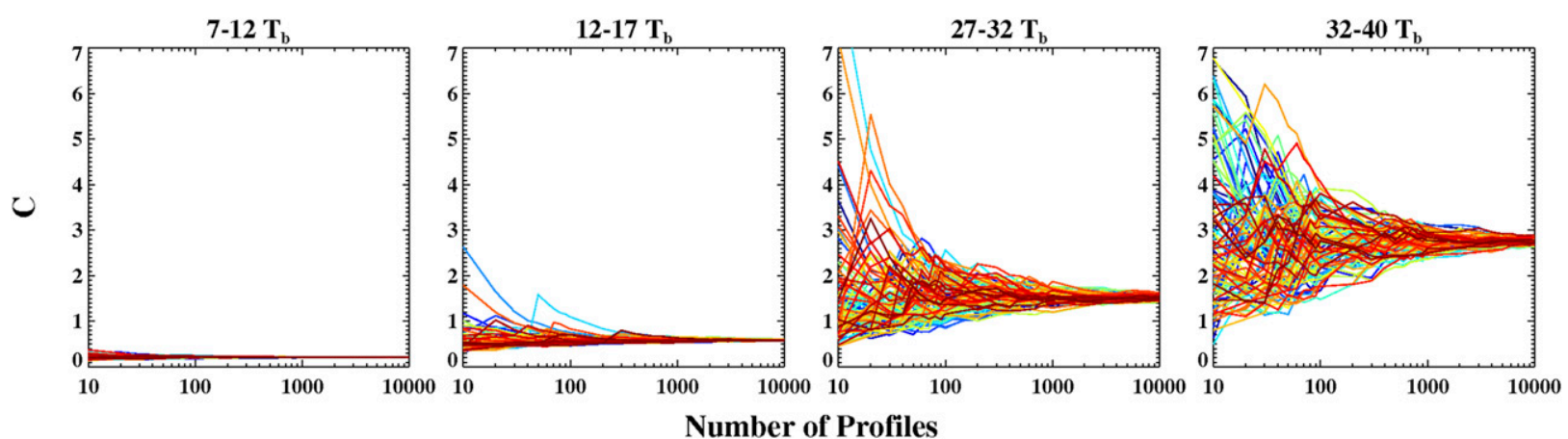

FIG. 4. As in Fig. 3, but for (left) $7-12 T_{b}$, (left center) $12-17 T_{b}$, (right center) $27-32 T_{b}$, and (right) $32-40 T_{b}$. 
TABLE 1. Errors in $C=L_{O} / L_{T}$ as a function of averaging, implied by Fig. 3 .

\begin{tabular}{ccc}
\hline \hline Averaging no. & Absolute RMS diff in $C$ & Percentage diff in $C$ \\
\hline 10 & 0.20 & $87.7 \%$ \\
100 & 0.17 & $66.2 \%$ \\
1000 & 0.05 & $18.7 \%$ \\
10000 & 0.015 & $5.8 \%$ \\
\hline
\end{tabular}

should be within about $19 \%$ of its true value; and determination of $C$ from 10000 profiles should be within less than $6 \%$ of its true value, all with $95 \%$ confidence. Furthermore, by examining results for the different stages of turbulence evolution, we expect these estimates to be robust. There are several factors involved in determining the actual value of $C$ for a turbulent layer. We expect different values of $C$ for different turbulence initiation mechanisms. Even for a given initiation mechanism, the value of $C$ will depend on the stage of the turbulence development. We have illustrated this for turbulence initiated by GW breaking. Thus, as mentioned previously, we expect greatly different values for $C$ for individual turbulence layers. By studying averaging as in Table 1, we hypothesize that such averaging will converge for the DNS results of Fritts et al. (2016), which contains different turbulence initiation mechanisms and different stages of turbulence evolution. This does appear to be the case if the turbulence cases include the variety of turbulence initiation mechanisms as well as the different stages of turbulence evolution. Table 1 is for the time span where the turbulence was in its active phase. We expect somewhat different results for other stages of turbulence development, but nonetheless the results of Table 1 still will serve as a useful guide.

Of course, it is not the value of $C$ in which we are primarily interested, but rather atmospheric turbulence parameters such as $\varepsilon$; therefore as a further check we have calculated averages of direct calculations of $\varepsilon$ from averages of $\varepsilon$ calculated by Thorpe analysis. More specifically, we have compared profile averages of $\varepsilon$, directly calculated from DNS "profiles" through the Fritts et al. (2016) results, with that calculated by Thorpe analysis using the $C$ that was converged upon for 10000 profiles for each of the time spans. Averages of these results from 10000 profiles through the Fritts et al. (2016) results indicate that, for $7 T_{b}<t<12 T_{b}$ (the period of developing turbulence), the average $\varepsilon$ as calculated by Thorpe analysis exceeds the direct calculation of $\varepsilon$ by a factor of 2.09. The analogous overestimate of $\varepsilon$ by Thorpe analysis for $12 T_{b}<t<27 T_{b}$ (the period of active turbulence) was a factor of 2.12; for $27 T_{b}<t<32 T_{b}$, a period for which turbulence is decaying, the overestimate is by a factor of 6.62 ; for
$32 T_{b}<t<37 T_{b}$, a period over which turbulence is further decaying, the overestimate was a factor of 9.34. Clearly then, there is a greater error in Thorpe analysis of $\varepsilon$ for more mature turbulence. This is as expected since the larger $C$ s are associated with more mature turbulence with lower values of $\varepsilon$, and applying these to regions of younger turbulence, characterized by lower $C$ s, overestimates $\varepsilon$.

\section{Conclusions}

In this brief paper, we have shown, using DNS modeling, that for GW breaking induced turbulence $C=L_{O} / L_{T}$ starts from zero, levels off at about 0.17 , and then increases to large values. We also anticipate that other sources of atmospheric turbulence, such as KHI and density intrusions, should behave similarly but likely level off at different values and with different durations of these intervals. The results of Fritts et al. (2016) suggest that the $C$ s for other sources of turbulence might level off at larger values than those for GW breaking.

Clearly, Thorpe analysis from single radiosonde profiles will not give quantitatively reliable values of the Ozmidov scale $L_{O}$ and thus will give unreliable values for the turbulence kinetic energy dissipation rate $\varepsilon$. We show by studying synthetic sounding profiles from the DNS modeling by Fritts et al. (2016) how averaging numbers of profiles gives more reliable values for $C$ and $\varepsilon$. This study implies that averaging 100 profiles should give reliable turbulence determinations for $C$ to about $65 \%$ with $95 \%$ confidence and that averaging 1000 profiles should give similarly reliable results to less than $20 \%$. Note that these results were obtained for the average value of $C$ with each profile, so that the averaging as a function of altitude will likely give somewhat larger errors for $C$ in the Thorpe analysis, with similarly somewhat larger errors for $\varepsilon$. Nonetheless, we believe that our estimates still may serve as a valuable guide to the reliability of profiles of $\varepsilon$.

An interesting implication of this study is that determining the value of $C$ at which it is nearly constant for a significant interval for each turbulence source type may provide a qualitative measure of the mixture of turbulence sources, which may be different in the troposphere and stratosphere and/or vary with time of day, and/or season, and/or geographic location. Researchers M. Kohma and K. Sato (2018, personal communication) have already obtained some preliminary results showing evidence for different $C$ values in the middle and upper troposphere than in the stratosphere, but more can be done on this subject. Another point is that our derived averaged $C$ values are representative of the DNS 
modeling of Fritts et al. (2016). This DNS modeling differs from the atmosphere in that the model is Boussinesq, and, once initiated, this DNS modeling has no continuing energy input. Improving the realism and diversity of initial conditions in DNS models of these dynamics will enable more confident explorations and comparisons with, and guidance of, atmospheric assessments of $L_{O}, L_{T}$, and $C$ and their implications.

This study of the convergence of $C$ suggests that a reason why the values of $C$ to which large number of profiles converge might be larger than the $C$ 's found at the point of their leveling off for individual instability sources of turbulence might be the following: when many profiles are sampled through an evolving turbulent environment, it is likely that many of these profiles will have encountered decaying turbulence, for which the $C$ 's tend to be large. In this decaying turbulence, $L_{T}$ would be small because of the turbulence smoothing out the gradients that led to the turbulence, but $L_{O}$ could still be sizable since the turbulence has not yet fully disappeared. This likely plays a large, and perhaps even dominant, role in making the domain-averaged values of $C$ in Fritts et al. (2016), larger than the leveling off values of $C$ for individual instability processes that initiate the turbulence. This also likely accounts for the relatively large values of $C$ in the lower panel of Fig. 14 in Fritts et al. (2016). Also, we might expect higher values of $C$ in environments with larger Reynolds numbers, since turbulence will decay more slowly, and remain stronger for longer times, in environments with higher Reynolds numbers, so that the probability of encountering layers with decaying turbulence will be higher in environments with larger Reynolds numbers. This might explain why the averaged values for $C$ (about 0.8) from Dillon (1982) might be relatively larger than those in averages for the atmosphere since turbulence dies out more slowly in the ocean than in the atmosphere. For instance, Clayson and Kantha (2008) used a value of $C=0.55$ for turbulence in the free atmosphere.

Papers by Mater et al. (2015) and Mashayek et al. (2017) have recently explored the factors that influence the values of $C=L_{O} / L_{T}$. The Mater et al. (2015) paper examined values of $C$ derived from measurements three different oceanic sites. They indicate that the value of $C$ decreases with increasing sizes of turbulent overturning. This is consistent with our results since large depths of overturning are associated with larger values of $L_{T}$, which tend to occur during the early stages of turbulence development from instabilities. Mashayek et al. (2017) performed high-resolution simulations that allowed following the time evolution of $L_{O}$ and $L_{T}$. They found that maximum turbulence mixing occurred when the value of $L_{O}$ is similar to that of $L_{T}$. This is also consistent with our results since we found that $L_{T} \gg L_{O}$ during the early development of turbulence, and $L_{T} \ll L_{O}$ during the turbulence decay stage.

The hypotheses made in this paper need to be explored further by more models of individual turbulenceinducing processes and more DNS-modeling studies, as well as comparison with observations of atmospheric turbulence.

Acknowledgments. This research was supported by National Science Foundation Grants AGS-15103545, AGS-1758293, AGS-1632772, and AFOSR FA955018-1-0009, and Dr. Joseph Werne of Colorado Research Associates in Boulder, Colorado, originally developed the TRIPLE code used to generate the DNS results used in this paper. We also found conversations with Dr. Werne to be valuable in the course of this research. We thank the anonymous reviewers whose constructive comments helped to improve the paper. We gratefully acknowledge access to large computational resources provided by the U.S. Department of Defense High Performance Computing Modernization Office to perform the DNS used in this study.

\section{REFERENCES}

Clayson, C. A., and L. Kantha, 2008: On turbulence and mixing in the free atmosphere inferred from high-resolution soundings. J. Atmos. Oceanic Technol., 25, 833-852, https://doi.org/ 10.1175/2007JTECHA992.1.

Dillon, T. M., 1982: Vertical overturns: A comparison of Thorpe and Ozmidov length scales. J. Geophys. Res., 87, 9601-9613, https://doi.org/10.1029/JC087iC12p09601.

Fritts, D. C., L. Wang, J. Werne, T. Lund, and K. Wan, 2009: Gravity wave instability dynamics at high Reynolds numbers. Part II: Turbulence evolution, structure, and anisotropy. J. Atmos. Sci., 66, 1149-1171, https://doi.org/10.1175/2008JAS2727.1.

$\longrightarrow,-$, M. A. Geller, D. A. Lawrence, J. Werne, and B. B. Balsley, 2016: Numerical modeling of multi-scale dynamics at a high Reynolds number: Instabilities, turbulence, and an assessment of Ozmidov and Thorpe scales. J. Atmos. Sci., 73, 555-578, https://doi.org/10.1175/JAS-D-14-0343.1.

Heisenberg, W., 1948: Zur statistischen Theorie der Turbulenz (On the statistical theory of turbulence). Z. Phys., 124, 628-657, https://doi.org/10.1007/BF01668899.

Kohma, M., K. Sato, Y. Tomikawa, K. Nishimura, and T. Sato, 2019: Estimate of turbulent energy dissipation rate from the VHF radar and radiosonde observations in the Antarctic. J. Geophys. Res. Atmos., 124, 2976-2993, https://doi.org/ 10.1029/2018JD029521.

Lübken, F.-J., 1992: On the extraction of turbulent parameters from atmospheric density fluctuations. J. Geophys. Res., 97, 20385-20395, https://doi.org/10.1029/92JD01916.

_ 1997: Seasonal variation of turbulent energy dissipation rates at high latitudes as determined by in situ measurements of neutral density fluctuations. J. Geophys. Res., 102, 13 441-13 456, https://doi.org/10.1029/97JD00853.

_ W. Hillert, G. Lehmacher, and U. von Zahn, 1993: Experiments revealing small impact of turbulence on the energy 
budget of the mesosphere and lower thermosphere. J. Geophys. Res., 98, 20369-20384, https://doi.org/10.1029/ 93JD02055.

Mashayek, A., C. P. Caulfield, and W. R. Peltier, 2017: Role of overturns in optimal mixing in stratified mixing layers. J. Fluid Mech., 826, 522-552, https://doi.org/10.1017/ jfm.2017.374.

Mater, B. D., S. K. Venayagamoorthy, L. St. Laurent, and J. N. Moum, 2015: Biases in Thorpe-scale estimates of turbulence dissipation. Part I: Assessments from large-scale overturns in oceanographic data. J. Phys. Oceanogr., 45, 2497-2521, https:// doi.org/10.1175/JPO-D-14-0128.1.

Schneider, A., M. Gerding, and F.-J. Lübken, 2015: Comparing turbulent parameters obtained from LITOS and radiosonde measurements. Atmos. Chem. Phys., 15, 2159-2166, https:// doi.org/10.5194/acp-15-2159-2015.

__ J. J. Wagner, J. Söder, M. Gerding, and F.-J. Lübken, 2017: Case study of wave breaking with high-resolution turbulence measurements with LITOS and WRF simulations. Atmos. Chem. Phys., 17, 7941-7954, https://doi.org/10.5194/ acp-17-7941-2017.
Scotti, A., 2015: Biases in Thorpe-scale estimates of turbulence dissipation. Part II: Energetics arguments and turbulence simulations. J. Phys. Oceanogr., 45, 2522-2543, https:// doi.org/10.1175/JPO-D-14-0092.1.

Smyth, W. D., and J. N. Moum, 2000: Length scales of turbulence in stably stratified mixing layers. Phys. Fluids, 12, 1327, https:// doi.org/10.1063/1.870385.

Tatarskii, V. I., 1971: The Effects of the Turbulent Atmosphere on Wave Propagation. Israel Program for Scientific Translations, $472 \mathrm{pp}$.

Thorpe, S. A., 1977: Turbulence and mixing in a Scottish loch. Philos. Trans. Roy. Soc. London, 286A, 125-181, https://doi.org/10.1098/ rsta.1977.0112.

Wilson, R., F. Dalaudier, and H. Luce, 2011: Can one detect small-scale turbulence from standard meteorological radiosondes? Atmos. Meas. Tech., 4, 795-804, https://doi.org/10.5194/ amt-4-795-2011.

, H. Luce, H. Hashiguchi, M. Shiotani, and F. Dalaudier, 2013: On the effect of moisture on the detection of tropospheric turbulence from in situ measurements. Atmos. Meas. Tech., 6, 697-702, https://doi.org/10.5194/amt-6-697-2013. 